Annals of Plant and Soil Research 23(2): 209-214 (2021)

https://doi.org/10.47815/apsr.2021.10059

\title{
Impact of cement industries dust on soil properties in Bhatapara,Chhattisgarh
}

\author{
ANURAG, RAHUL KUMAR* AND NEELKAMAL SAHU \\ Department of Soil Science and Agricultural Chemistry, IGKV, Raipur Chhattisgarh
}

Received: March, 2021; Revised accepted: April, 2021

\begin{abstract}
The present investigation was carried around cement industries at Bhatapara during 2017-18, to study the different soil properties as affected by the dust of cement Industries in Bhatapara Chhattisgarh. Two hundred fifty six composite soil samples were taken from around the cement industries i.e., from eight radiant wind directions viz., North, South, East, West, Northeast, Northwest, Southeast and Southwest in clockwise manner at the distances $0.5,1,2$, and $3 \mathrm{~km}$ from the surface $(0-15 \mathrm{~cm})$ and sub-surface $(15-30 \mathrm{~cm})$ soil depths. Statistical analysis was done in 3-factors factorial designed experiment using CRD and the effect of cement dust on soil properties were also correlated with wind directions $\left(X_{1}\right)$, distances $\left(X_{2}\right)$ and soil depths $\left(X_{3}\right)$. Thephysico-properties of soils showed a significant increase in sand and silt in south-west and west wind directions. Significant increase in clay content was also observed in west and south-wind direction. An increase in $\mathrm{pH}$, electrical conductivity and calcium carbonate content in soil $(0-15 \mathrm{~cm})$ soil up to $0.5 \mathrm{~km}$ distance in the southwest wind direction was also observed. Organic carbon content in soils also increased significantly with increase in distance at surface soilin the southwest wind direction.
\end{abstract}

Keywords: Cement dust, $\mathrm{pH}, \mathrm{EC}$, organic carbon and $\mathrm{CaCO}_{3}$.

\section{INTRODUCTION}

Cement dust is a mixture of $\mathrm{Ca}, \mathrm{K}, \mathrm{Si}$ and $\mathrm{Na}$ which often include heavy metals like As, Al, $\mathrm{Cd}, \mathrm{Pb}, \mathrm{Zn}, \mathrm{Fe}$, and $\mathrm{Cr}$. Majority of these elements in excess amounts are potentially harmful to the biotic and a biotic components of the environment (Gbadebe and Bankole, 2007). The cement particles get deposited on top soil and vegetation as well as enter into the soil as dry, humid or occult deposits and can affect its physicochemical properties (Gupta and Sharma, 2012). The cement dust which is highly alkaline give rise to high $\mathrm{pH}$ values (Mlitanet al., 2013). Some of the metals associated with cement dust, such as sodium, potassium, copper, zinc, calcium, magnesium, manganese and iron are necessary for microbial growth and metabolism, but can become toxic if their concentration exceeds certain threshold (Asadu and Agada, 2008). Air pollutants generated by the cement manufacturing process consist primarily of alkaline particulates from the raw and finished materials. The direct effects of cement dust pollution are the alkalization of the ecosystem and the changing of the chemical composition of soils. The main impacts of the cement activity on the environment are the broadcasts of dusts and gases. The pollutant particles can enter into soil as dry, humid or occult deposits and can undermine its physicochemical properties. Thus, cement dust pollution has a negative effect on the physico-chemical properties and the biological activity of the soil. Soil microbial activity is important for the nutrient biogeochemical cycling and it is negatively affected by the cement dust pollution (Ocakand Nowak, 2004). Soil physico-chemical and biological properties are affected by the type of parent materials that formed the soil or various human activities, such as farming, depositions of industrial wastes, and so forth. Cement dust is one of such industrial wastes that affect the soil quality.

\section{MATERIALS AND METHODS}

The experimental site for soil sampling was selected in surrounding area of Ambuja Cement Industries, Rawan, which is situated about $16 \mathrm{~km}$ from Bhatapara town of district Balodabazar-bhatapara and $65 \mathrm{~km}$ from state capital Raipur in Chhattisgarh. District lies in $21^{\circ} 3248^{\prime \prime}$ north and $81^{\circ} 5640^{\prime \prime}$ east. It has an average elevation of $254 \mathrm{~m}(833 \mathrm{ft})$ in Chhattisgarh state.The study area around the cement plant was divided into eight radiant directions of north, south, east, west, northeast,

*Author for Correspondence: E-mail: rahulsoiligkv@gmail.com

Assistant ProfessorSoil Science and Agricultural Chemistry, COA Bharatpur 
northwest, southeast and southwest in clockwise manner. Soil samples were taken in the monthsof June- July 2017 with the help of soil auger at surface $(0-15 \mathrm{~cm})$ and sub-surface (15$30 \mathrm{~cm}$ ) depth at the distances $0.5,1,2$, and 3 $\mathrm{km}$. (8 samples each)from cement plant and collected in plastic bags and analyzed for $\mathrm{pH}$ by glass electrode $\mathrm{pH}$ meter taking 1:2.5 soil watersuspensions (Jackson 1973).Electrical conductivity was determined by taking supernatant liquid of soil water suspension by using electrical conductivity and organic carbon was determined by Walkley and Black rapid titration method as described by Jackson (1973) the calcium carbonate content was determined by rapid titration method and soil texture determined by International Pipette Method.

\section{RESULTS AND DISCUSSION}

\section{Soil texture}

The sand content in soils varied from 10.7 to $30.3 \%$ with respect to wind direction, distances from industries and soil depth (Table 1). The lowest and highest per cent sand content was recorded 10.7 and $27.5 \%$ and 13.3 and 30.3 $\%$ in soils at surface $(0-15 \mathrm{~cm})$ and sub-surface $(15-30 \mathrm{~cm})$ soil at 3 and $0.5 \mathrm{~km}$ distances in relation to south and west wind directions, respectively. Soil particle sizesand $(Y)$ content in soils was positively and significantly correlated with the distances from industries $\left(X_{2}\right)$ and negatively and significantly with soil depth $\left(X_{3}\right)$ for all the wind directions. If the wind direction $\left(X_{1}\right)$ and distances from industries $\left(X_{2}\right)$ are fixed, then the soil particle size $(Y)$ increased with the increasing soil depth. Similarly, if the wind direction and soil depth are fixed, then the soil particle sizes $(\mathrm{Y})$ significantly decreased with the increasing distances from industries. The silt content in soils varied from 27.3 to $44.1 \%$ with respect to wind direction, distances and soil depth. The lowest and highest silt content was recorded 28.0 and 44.1 and 27.3 and $41.3 \%$ in soil at surface $(0-15 \mathrm{~cm})$ and sub surface $(15-30$ $\mathrm{cm})$ soil at distances 3 and $2 \mathrm{~km}$ in relation to southwest and west wind directions, respectively (Table 1). Soil particle sizesilt $(Y)$ content in soils was positively significantly correlated with the distances from industries $\left(X_{2}\right)$ and negatively and significantlywith soil depth $\left(X_{3}\right)$ for the all wind directions under study. If the wind direction $\left(X_{1}\right)$ and distances from industries $\left(X_{2}\right)$ are fixed, then the soil particle sizesilt $(Y)$ decreased with the increasing soil depth. Similarly, if the wind direction $\left(X_{1}\right)$ and soil depth $\left(X_{3}\right)$ are fixed, then the soil particle sizesilt $(Y)$ significantly increased with the increasing distances from industries.

The clay content in soils varied from 29.3 to $57.3 \%$ with respect to wind direction, distances and soil depth. The lowest and highest clay content was recorded 30.1 and 57.3 and 29.3 and $55.3 \%$ in soils at surface $(0-15 \mathrm{~cm})$ and sub-surface $(15-30 \mathrm{~cm})$ soil at distances 0.5 and $3 \mathrm{~km}$ in relation to west and southwest wind directions respectively (Table 1 ). Soil particle sizeclay $(\mathrm{Y})$ content in soils was positively and significantly correlated with the distances from industries $\left(X_{2}\right)$ and negatively and significantly with soil depth $\left(X_{3}\right)$ for all the wind directions. If the wind direction $\left(X_{1}\right)$ and distances from industries $\left(X_{2}\right)$ are fixed, then the soil particle sizeclay $(Y)$ decreased with the increasing soil depth. Similarly, if the wind direction $\left(X_{1}\right)$ and soil depth $\left(X_{3}\right)$ are fixed, then the soil clay $(Y)$ significantly increases with the increasing distances from industries. The highest per cent sand in Ulfisol was recorded $30.3 \%$ in subsurface $(15-30 \mathrm{~cm})$ soil at $0.5 \mathrm{~km}$ distance in relation to west wind directions. The highest per cent silt content in Ulfisol was recorded $44.1 \%$ in soil at surface $(0-15 \mathrm{~cm})$ soil depth at distances $3 \mathrm{~km}$ in relation to west wind directions. The highest per cent clay in Vertisol was recorded $57.3 \%$ in soils at surface $(0-15$ $\mathrm{cm}$ ) soil depth at distances $3 \mathrm{~km}$ in relation to southwest wind directions. 
Table 1: Effect of wind directions (A), distances from industries $(B)$ and soil depths $(C)$ on particle sand, silt and clay $\%$ in soils

\begin{tabular}{|c|c|c|c|c|c|c|c|c|}
\hline \multirow{4}{*}{$\begin{array}{c}\text { Wind } \\
\text { Directions }(A)\end{array}$} & \multicolumn{8}{|c|}{ Distances From Industries (B) Sand } \\
\hline & \multicolumn{2}{|c|}{$0.5 \mathrm{~km}$} & \multicolumn{2}{|c|}{$1 \mathrm{~km}$} & \multicolumn{2}{|c|}{$2 \mathrm{~km}$} & \multicolumn{2}{|c|}{$3 \mathrm{~km}$} \\
\hline & \multicolumn{8}{|c|}{ Soil Depths (C) } \\
\hline & Surface & $\begin{array}{c}\text { Sub- } \\
\text { surface }\end{array}$ & Surface & $\begin{array}{c}\text { Sub- } \\
\text { surface }\end{array}$ & Surface & $\begin{array}{c}\text { Sub- } \\
\text { surface }\end{array}$ & Surface & $\begin{array}{c}\text { Sub- } \\
\text { surface }\end{array}$ \\
\hline North & $23.5_{D}^{\mathrm{e}} 1$ & $25.4^{d}{ }^{d} 2$ & $22.6^{\mathrm{e}}{ }_{\mathrm{c}} 1$ & $25.0^{\mathrm{e}} \mathrm{E}$ & $21.6^{d}{ }_{B} 1$ & $23.3^{C}{ }^{C} 2$ & $20.6^{d}{ }_{A} 1$ & $22.6^{d}{ }^{d} 2$ \\
\hline South & $13.2^{\mathrm{a}}{ }_{\mathrm{C}} 1$ & $15.5^{\mathrm{a}} \mathrm{2}$ & $13.2^{\mathrm{a}}{ }_{\mathrm{C}} 1$ & $15.3^{\mathrm{a}} \mathrm{E}^{2}$ & $12.2^{\mathrm{a}}{ }_{\mathrm{B}} 1$ & $14.6^{\mathrm{a}}{ }^{2} 2$ & $10.7_{\mathrm{A}}^{\mathrm{a}} 1$ & $13.3^{\mathrm{a}}{ }_{\mathrm{c}} 2$ \\
\hline East & $23.1^{d}{ }_{D} 1$ & $26.3^{\mathrm{e}}{ }_{\mathrm{G}} 2$ & $22.2^{\mathrm{d}}{ }_{\mathrm{C}} 1$ & $24.4^{\mathrm{d}} \mathrm{F} 2$ & $21.7^{\mathrm{d}}{ }_{\mathrm{B}} 1$ & $23.5^{\mathrm{c}} \mathrm{E} 2$ & $20.3_{A}^{d} 1$ & $22.3^{d}{ }_{c} 2$ \\
\hline West & $27.5^{\mathrm{n}} 1$ & $30.3_{G}^{n} 2$ & $26.3^{n}{ }_{c} 1$ & $28.2^{\mathrm{n}} \mathrm{E} 2$ & $25.3^{\mathrm{g}} 1$ & $27.6_{D}^{\dagger} 2$ & $24.3^{\mathrm{g}} 1$ & $28.5_{\mathrm{F}}^{\dagger} 2$ \\
\hline NE & $24.1_{D}^{\dagger} 1$ & $27.3_{G}^{\dagger} 2$ & $23.6_{C}^{\dagger} 1$ & $26.3_{F}^{\dagger} 2$ & $22.2^{\mathrm{e}} 1$ & $25.5^{\mathrm{d}} 2$ & $21.3_{\mathrm{A}}^{\mathrm{e}} 1$ & $24.2^{\mathrm{e}}{ }^{2} 2$ \\
\hline NW & $25.6^{g}{ }_{D} 1$ & $28.2^{g}{ }_{G} 2$ & $24.4^{g}{ }_{C} 1$ & $27.3^{g} \mathrm{~F} 2$ & $23.5_{B}^{\dagger} 1$ & $26.5^{\mathrm{e}} \mathrm{E} 2$ & $22.5_{A}^{\dagger} 1$ & $24.5^{\mathrm{e}}{ }^{2} 2$ \\
\hline SE & $15.3^{\mathrm{b}}{ }_{\mathrm{D}} 1$ & $17.5^{\mathrm{b}}{ }_{\mathrm{G}} 2$ & $14.5^{\mathrm{b}}{ }_{\mathrm{C}} 1$ & $16.5^{\mathrm{b}} \mathrm{E}^{2}$ & $13.7^{\mathrm{b}}{ }_{\mathrm{B}} 1$ & $15.4^{\mathrm{b}}{ }^{\mathrm{b}} 2$ & $12.6^{b}{ }_{A} 1$ & $14.3^{b}{ }_{c} 2$ \\
\hline SW & $16.8^{\mathrm{C}}{ }_{\mathrm{D}} 1$ & $19.9^{c}{ }^{c} 2$ & $16.2^{c}{ }_{C} 1$ & $19.2^{c}{ }_{G} 2$ & $15.4^{\mathrm{C}}{ }_{\mathrm{B}} 1$ & $18.4^{\mathrm{C}} \mathrm{F} 2$ & $14.5^{c}{ }_{A} 1$ & $17.3^{\mathrm{C}}{ }_{\mathrm{E}} 2$ \\
\hline \multirow[t]{2}{*}{ AXBXC } & & & SEm \pm & .12 & $C D-5^{\circ}$ & 0.34 & & \\
\hline & \multicolumn{8}{|c|}{ Silt \% } \\
\hline North & $40.4^{d}{ }^{d} 2$ & $39.3_{A}^{\dagger} 1$ & $40.6^{\mathrm{e}}{ }^{2} 2$ & $39.9^{\dagger} 1$ & $41.6_{\mathrm{E}}^{\dagger} 2$ & $40.2^{\dagger}{ }^{\dagger} 1$ & $42.4_{\mathrm{F}}^{\mathrm{e}} 2$ & $40.5_{D}^{\dagger} 1$ \\
\hline South & $36.1^{\mathrm{C}}{ }_{\mathrm{F}} 2$ & $35.8^{\mathrm{C}} 1$ & $35.2^{\mathrm{C}}{ }^{\mathrm{D}} 2$ & $35.0^{\mathrm{c}}{ }_{\mathrm{C}} 1$ & $35.2^{c}{ }^{c} 2$ & $34.9^{\mathrm{C}}{ }_{\mathrm{B}} 1$ & $34.8^{\mathrm{C}}{ }_{\mathrm{B}} 2$ & $34.3^{\mathrm{C}}{ }_{\mathrm{A}} 1$ \\
\hline East & $40.2^{d}{ }^{d} 2$ & $38.2^{d}{ }_{A} 1$ & $40.3^{d}{ }^{d} 2$ & $38.6^{d}{ }_{B} 1$ & $41.1^{d}{ }^{d} 2$ & $39.4_{C}^{e} 1$ & $41.3^{d}{ }^{d} 2$ & $39.6^{d}{ }_{c} 1$ \\
\hline West & $42.2^{g}{ }^{g} 2$ & $40.3^{g}{ }_{A} 1$ & $42.4^{g}{ }^{g} 2$ & $40.5^{\mathrm{g}}{ }_{\mathrm{B}} 1$ & $43.1^{\mathrm{n}} \mathrm{E} 2$ & $41.3^{g}{ }^{1}$ & $44.1_{\mathrm{F}}^{\dagger} 2$ & $40.2^{\mathrm{e}}{ }_{\mathrm{A}} 1$ \\
\hline NE & $41.2_{C}^{\dagger} 2$ & $39.3_{A}^{\dagger} 1$ & $41.4_{D}^{\dagger} 2$ & $39.4_{A}{ }_{A} 1$ & $42.3^{g}{ }^{g} 2$ & $39.3^{d}{ }_{A} 1$ & $42.4^{\mathrm{e}} \mathrm{E} 2$ & $40.3^{\mathrm{e}}{ }_{\mathrm{B}} 1$ \\
\hline NW & $40.4^{\mathrm{e}}{ }^{\mathrm{C}} 2$ & $38.6^{\mathrm{e}}{ }_{\mathrm{A}} 1$ & $40.5^{\mathrm{e}}{ }^{2} 2$ & $38.7_{A}^{d} 1$ & $41.4^{\mathrm{e}}{ }^{\mathrm{E}} 2$ & $39.2^{d}{ }_{B} 1$ & $42.3^{\mathrm{e}} \mathrm{F} 2$ & $40.7^{g}{ }_{D} 1$ \\
\hline SE & $32.3^{b}{ }^{b} 2$ & $32.1_{B}^{b} 1$ & $32.8^{b}{ }^{b} 2$ & $32.6^{b}{ }_{D} 1$ & $32.9^{b}{ }^{b} 2$ & $32.8^{\mathrm{b}}{ }_{\mathrm{D}} 1$ & $31.5^{\mathrm{b}}{ }_{\mathrm{D}} 2$ & $31.5^{b}{ }_{A} 1$ \\
\hline SW & $28.6^{a}{ }^{a} 2$ & $27.5^{a}{ }_{B} 1$ & $29.0^{\mathrm{a}} \mathrm{F} 2$ & $28.3^{a}{ }_{D} 1$ & $29.2^{\mathrm{a}}{ }_{\mathrm{G}} 2$ & $28.1^{\mathrm{a}}{ }_{\mathrm{C}} 1$ & $28.0^{a}{ }^{a} 2$ & $27.3^{\mathrm{a}}{ }_{\mathrm{A}} 1$ \\
\hline \multirow[t]{2}{*}{ AXBXC } & & & SEm \pm 0 & & CD- & 0.17 & & \\
\hline & \multicolumn{8}{|c|}{ Clay \% } \\
\hline North & $36.0 \mathrm{dC2}$ & $35.2 \mathrm{~dB} 1$ & 36.7dE2 & $35.0 \mathrm{dA} 1$ & 36.7dE2 & 36.4dD1 & $36.9 \mathrm{dF} 2$ & $36.7 \mathrm{dE} 1$ \\
\hline South & $50.5 \mathrm{eC} 2$ & $48.6 \mathrm{eA} 1$ & 51.5fE2 & 49.6fB1 & $52.4 \mathrm{fF} 2$ & $50.4 \mathrm{fC} 1$ & 54.3fG2 & $52.2 \mathrm{fF} 1$ \\
\hline East & $36.1 \mathrm{~dB} 2$ & $35.4 \mathrm{dA} 1$ & 37.3eE2 & $36.9 \mathrm{eC} 1$ & $37.1 \mathrm{eD} 2$ & $36.9 \mathrm{eC} 1$ & 38.3eG2 & $37.9 \mathrm{eF} 1$ \\
\hline West & $30.1 \mathrm{aB} 2$ & 29.3aA1 & $31.2 \mathrm{aC} 2$ & $31.1 \mathrm{aC} 1$ & 31.4aD2 & $31.0 \mathrm{aC} 1$ & 31.4aD2 & $31.1 \mathrm{aC} 1$ \\
\hline NE & $34.5 \mathrm{cC} 2$ & 33.3cA1 & $34.9 \mathrm{bD} 2$ & $34.1 \mathrm{cB} 1$ & $35.4 \mathrm{cF} 2$ & 35.1cE1 & 36.1cG2 & 35.3cF1 \\
\hline NW & 33.9bB2 & 33.0bA1 & 35.1cE2 & 33.9bB1 & 35.0bE2 & $34.2 \mathrm{bC} 1$ & 35.0bE2 & 34.6bD1 \\
\hline SE & $52.2 f D 2$ & $50.2 \mathrm{fA} 1$ & $52.6 \mathrm{gE} 2$ & $50.7 \mathrm{gB} 1$ & $53.2 \mathrm{gF} 2$ & $51.7 \mathrm{gC} 1$ & $55.7 \mathrm{gH} 2$ & $54.0 \mathrm{gG} 1$ \\
\hline SW & $54.5 \mathrm{gC} 2$ & $52.5 \mathrm{gA} 1$ & $54.6 \mathrm{hC2}$ & 52.3hA1 & $55.2 \mathrm{hD} 2$ & 53.3hB1 & 57.3hE2 & 55.3hD1 \\
\hline AXBXC & \multicolumn{4}{|c|}{ SEm \pm 0.07} & \multicolumn{2}{|c|}{ CD-5\% 0.2} & & \\
\hline
\end{tabular}

\section{Soil reaction $(\mathrm{pH})$}

The $\mathrm{pH}$ content in soils varied from 6.55 to 7.58 at the distances 3 and $0.5 \mathrm{~km}$ from industries site in relation to east to southwest wind directions, respectively (Table 2). Significant differences in soil $\mathrm{pH}$ were reported with respect to all the four distances viz. 0.5, 1,2 and $3 \mathrm{~km}$ and with each fixed wind directions. Soil $\mathrm{pH}(\mathrm{Y})$ content in soils was negativelyand significantly correlated with the distances from industries $\left(\mathrm{X}_{2}\right)$ for all the wind directions. If the wind directions $\left(X_{1}\right)$ are fixed, then the soil $\mathrm{pH}$
(Y) decreased with the increasing distances from industries. The higher values of $\mathrm{pH}$ content noted in soils at the distances of 0.5 and $1 \mathrm{~km}$ as compared to distances of 2 and $3 \mathrm{~km}$ in relation to southwest wind direction if may be due to high level of dust deposition and as most of the $\mathrm{Ca}$ in the cement dust in the form of oxide, hydroxide and carbonate formed lime that increased the soil $\mathrm{pH}$. Similar findings were reported by Ahiamadjie et al. (2011) and Raajasubramanianet al. (2011). 
Table 2: Effect of wind directions (A) and distances from industries (B) on soils $\mathrm{pH}$

\begin{tabular}{|c|c|c|c|c|}
\hline \multirow{2}{*}{ Wind Directions (A) } & \multicolumn{4}{|c|}{ Distances From Industries (B) } \\
\hline & $0.5 \mathrm{~km}$ & $1 \mathrm{~km}$ & $2 \mathrm{~km}$ & $3 \mathrm{~km}$ \\
\hline North & $7.01^{b} \mathrm{D}$ & $6.95^{b} \mathrm{C}$ & $6.86^{b}{ }_{B}$ & $6.76^{\mathrm{DA}}$ \\
\hline South & $7.25^{\mathrm{e}} \mathrm{D}$ & $7.15^{\mathrm{e}} \mathrm{c}$ & $7.05^{\mathrm{e}}{ }_{\mathrm{B}}$ & $6.88^{\mathrm{CA}}$ \\
\hline East & $6.78^{\mathrm{a}}{ }_{\mathrm{D}}$ & $6.70^{\mathrm{a}}{ }_{C}$ & $6.68^{\mathrm{a}}{ }_{\mathrm{B}}$ & $6.55^{\mathrm{aA}}$ \\
\hline West & $7.18^{\mathrm{d}} \mathrm{D}$ & $7.08^{\mathrm{d}}{ }_{\mathrm{C}}^{\mathrm{c}}$ & $6.98^{a^{\mathrm{b}}}$ & $6.85^{\mathrm{dA}}$ \\
\hline $\mathrm{NE}$ & $7.06^{\mathrm{C}} \mathrm{D}$ & $7.00^{\mathrm{C}} \mathrm{C}$ & $6.85^{\mathrm{b}}{ }_{\mathrm{B}}^{\mathrm{b}}$ & $6.78^{\mathrm{DA}}$ \\
\hline NW & $7.08^{\mathrm{C}} \mathrm{D}$ & $7.01^{\mathrm{c}} \mathrm{C}$ & $6.90^{\mathrm{C}}{ }_{\mathrm{B}}$ & $6.78^{\mathrm{bA}}$ \\
\hline SE & $7.38^{\dagger}$ & $7.25_{\mathrm{c}}^{\dagger}$ & $7.06^{\mathrm{e}_{\mathrm{B}}^{\mathrm{b}}}$ & $6.96^{\mathrm{dA}}$ \\
\hline SW & $7.58^{\mathrm{g}} \mathrm{D}$ & $7.48^{\mathrm{g}} \mathrm{C}$ & $7.38_{B}^{\dagger}$ & $7.25^{\mathrm{eA}}$ \\
\hline$A \times B$ & SEm \pm 0.019 & CD-5\% 0.053 & & \\
\hline
\end{tabular}

\section{Electrical conductivity (EC)}

Overall variations in the electrical conductivity were recorded from 0.15 to 0.33 $\mathrm{dSm}^{-1}$ with respects to wind directions, distances and soil depths (Table 3). The lowest and highest electrical conductivity content was recorded 0.16 and $0.33 \mathrm{dSm}^{-1}$ and 0.15 and 0.32 $\mathrm{dSm}^{-1}$ in soil at surface $(0-15 \mathrm{~cm})$ and subsurface $(15-30 \mathrm{~cm})$ soil at distances 3 and $0.5 \mathrm{~km}$ in relation to east to southwest wind directions, respectively. Electrical conductivity $(\mathrm{Y})$ in soils was negatively and significantly correlated with the distances from industries $\left(X_{2}\right)$ and soil depth $\left(X_{3}\right)$ for all the wind directions. If the wind direction $\left(\mathrm{X}_{1}\right)$ and distances from industries $\left(\mathrm{X}_{2}\right)$ are fixed, then the soil electrical conductivity $(\mathrm{Y})$ decreased with the increasing soil depth. Similarly, if the wind direction $\left(\mathrm{X}_{1}\right)$ and soil depth $\left(X_{3}\right)$ are fixed, then the soil electrical conductivity $(Y)$ significantly decreased with the increasing distances from industries. The electrical conductivityincreased significantly with decreasing distances and the higher values were recorded at $0.5 \mathrm{~km}$ distances in surface soil in relation to southwest wind direction which may be due to higher deposition of cement dust and its ingredients increased the soluble salt concentration. Similar findings were also reported by Ahiamadjieet al.(2011)and Raajasubramanianet al.(2011).

Table 3: Effect of wind directions (A), distances from industries (B) and soil depths (C) on electrical conductivity $\left(\mathrm{dSm}^{-1}\right)$ in soils

\begin{tabular}{|c|c|c|c|c|c|c|c|c|}
\hline \multirow{4}{*}{$\begin{array}{c}\text { Wind } \\
\text { Directions }(A)\end{array}$} & \multicolumn{8}{|c|}{ Distances From Industries (B) } \\
\hline & \multicolumn{3}{|c|}{$0.5 \mathrm{~km}$} & $1 \mathrm{~km}$ & & $2 \mathrm{~km}$ & & $3 \mathrm{~km}$ \\
\hline & \multicolumn{8}{|c|}{ Soil Depths (C) } \\
\hline & Surface & Sub-surface & Surface & Sub-surface & Surface & Sub-surface & Surface & Sub-surface \\
\hline North & $0.24^{\mathrm{b}}$ & $0.21^{\mathrm{b}}$ & $0.21^{\mathrm{b}}$ & $0.19^{\mathrm{b}}$ & $0.19^{\mathrm{b}}$ & $0.17^{\mathrm{b}}$ & $0.17^{\mathrm{b}}$ & $0.15^{\mathrm{a}}$ \\
\hline South & $0.30^{\mathrm{e}}$ & $0.27^{\mathrm{e}}$ & $0.28^{\mathrm{d}}$ & $0.27^{d}$ & $0.27^{\dagger}$ & $0.25^{\dagger}$ & $0.25^{\mathrm{e}}$ & $0.22^{\mathrm{e}}$ \\
\hline East & $0.21^{\mathrm{a}}$ & $0.19^{\mathrm{a}}$ & $0.19^{\mathrm{a}}$ & $0.17^{\mathrm{a}}$ & $0.17^{\mathrm{a}}$ & $0.15^{\mathrm{a}}$ & $0.16^{\mathrm{a}}$ & $0.15^{\mathrm{a}}$ \\
\hline West & $0.26^{\mathrm{d}}$ & $0.24^{d}$ & $0.25^{\mathrm{C}}$ & $0.23^{c}$ & $0.21^{\mathrm{c}}$ & $0.20^{c}$ & $0.21^{\mathrm{c}}$ & $0.18^{\mathrm{b}}$ \\
\hline $\mathrm{NE}$ & $0.25^{\mathrm{c}}$ & $0.23^{\mathrm{c}}$ & $0.25^{\mathrm{C}}$ & $0.23^{\mathrm{c}}$ & $0.23^{\mathrm{d}}$ & $0.21^{\mathrm{d}}$ & $0.21^{\mathrm{c}}$ & $0.19^{c}$ \\
\hline NW & $0.26^{\mathrm{d}}$ & $0.23^{\mathrm{c}}$ & $0.25^{\mathrm{c}}$ & $0.23^{\mathrm{c}}$ & $0.24^{e}$ & $0.22^{\mathrm{e}}$ & $0.23^{\mathrm{d}}$ & $0.20^{\mathrm{a}}$ \\
\hline SE & $0.31^{\dagger}$ & $0.29^{\dagger}$ & $0.29^{\mathrm{e}}$ & $0.27^{d}$ & $0.28^{\mathrm{g}}$ & $0.26^{g}$ & $0.27^{\dagger}$ & $0.24^{\dagger}$ \\
\hline SW & $0.33^{9}$ & $0.32^{g}$ & $0.31^{\dagger}$ & $0.29^{\mathrm{e}}$ & $0.29^{h}$ & $0.27^{\mathrm{h}}$ & $0.28^{g}$ & $0.25^{9}$ \\
\hline$A \times B$ & & & SEm \pm 0 & .002 & & CD-5\% 0.006 & & \\
\hline
\end{tabular}

\section{Organic carbon}

The organic carbon content varied from 2.4 to $5.0 \mathrm{~g} \mathrm{Kg}^{-1}$ the highest organic carbon value is recorded at $3 \mathrm{~km}$ distances while the lowest was recorded at $0.5 \mathrm{~km}$ distances in relation to south west andeast wind directions, respectively (Table 4). Significant differences in soil organic carbon were recorded with respect to all the four distances and with each fixed wind directions.Organiccarbon $(\mathrm{Y})$ content in soils was significantly and positively correlated with the 
distances from industries $\left(X_{2}\right)$ for all wind directions. If the wind directions $\left(X_{1}\right)$ are fixed, then the soil organic carbon $(Y)$ significantly increased with the increasing distances from industries. The organic carbon content in soil increased with increasing distances and recorded higher values at 2and $3 \mathrm{~km}$ as compared to nearer distances i.e. 0.5 and $1 \mathrm{~km}$ distances in relation to southwest to east wind direction. Cement dust alkalinity had negatively affect an organic carbon. Similar findings were reported by Grantzet al. (2008) and Pandey et al. (2011).

Table 4: Effect of wind directions (A) and distances from industries (B) on organic carbon $\mathrm{gkg}^{-1}$ ) in soil

\begin{tabular}{|c|c|c|c|c|}
\hline \multirow{2}{*}{ Wind Directions $(A)$} & \multicolumn{4}{|c|}{ Distances From Industries (B) } \\
\hline & $0.5 \mathrm{~km}$ & $1 \mathrm{~km}$ & $2 \mathrm{~km}$ & $3 \mathrm{~km}$ \\
\hline North & $2.8^{\mathrm{b}}{ }_{\mathrm{A}}$ & $3.1^{\mathrm{b}} \mathrm{B}$ & $3.4^{\mathrm{b}} \mathrm{C}$ & $3.9_{\mathrm{D}}^{\mathrm{b}}$ \\
\hline South & $3.8_{A}^{9}$ & $4.2^{g^{D}}$ & $4.6^{9} \mathrm{C}$ & $4.7^{\mathrm{e}}$ \\
\hline East & $2.4^{a}{ }_{A}$ & $2.9^{\mathrm{a}}{ }_{\mathrm{B}}$ & $3.1^{\mathrm{a}} \mathrm{C}$ & $3.4^{\mathrm{a}} \mathrm{D}$ \\
\hline West & $3.1_{\mathrm{A}}^{\mathrm{e}}$ & $3.4_{B}^{a}$ & $3.7^{a^{c}} \mathrm{c}$ & $4.1^{\mathrm{d}} \mathrm{D}$ \\
\hline NE & $2.9^{c_{A}^{A}}$ & $3.2^{\mathrm{C}}{ }_{\mathrm{B}}^{\mathrm{B}}$ & $3.6^{c}{ }_{C}^{c}$ & $4.0^{\mathrm{C}}$ \\
\hline NW & $3.0^{a_{A}}$ & $3.6^{\mathrm{e}}{ }_{\mathrm{B}}$ & $3.8^{e} \mathrm{C}$ & 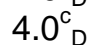 \\
\hline SE & $3.6_{A}^{\dagger}$ & $3.9_{\mathrm{B}}^{\dagger^{\circ}}$ & $4.2_{C}^{\dagger}$ & $4.7^{\mathrm{e}} \mathrm{D}$ \\
\hline SW & $4.0^{h_{A}^{n}}$ & $4.3^{\mathrm{h}}{ }_{B}$ & $4.7^{\mathrm{n}} \mathrm{C}$ & $5.0_{D}^{\dagger}$ \\
\hline AXB & SEm \pm 0.002 & CD- $5 \% \quad 0.0$ & & \\
\hline
\end{tabular}

\section{Calcium carbonate}

The content calcium carbonate in soils ranged from24.6 to $48.7 \mathrm{gkg}^{-1}$ at the distances 3 and $0.5 \mathrm{~km}$ in relation to east to southwest wind directions, respectively (Table 5). Significant differences in calcium carbonate content were recorded with respect to all the four distances and with each fixed wind directions.Calcium carbonate $(Y)$ content in soils were negatively and significantly correlated with the distances from industries $\left(X_{2}\right)$ for all the wind directions. If the wind directions $\left(X_{1}\right)$ are fixed, then the soil $\mathrm{CaCO}_{3}(Y)$ significantly decreased with the increasing distances from industries.

Table 5: Effect of wind directions (A), distances from industries (B) and soil depth (C) on $\mathrm{CaCO}_{3}(\mathrm{~g}$ $\mathrm{kg}^{-1}$ ) in soils

\begin{tabular}{|c|c|c|c|c|c|c|}
\hline \multirow{2}{*}{ Wind Directions $(\mathrm{A})$} & \multicolumn{4}{|c|}{ Distances From Industries (B) } & \multicolumn{2}{|c|}{ Soil Depths ( C ) } \\
\hline & $0.5 \mathrm{~km}$ & $1 \mathrm{~km}$ & $2 \mathrm{~km}$ & $3 \mathrm{~km}$ & $0-15 \mathrm{~cm}$ & $15-30 \mathrm{~cm}$ \\
\hline North & $38.0^{\mathrm{b}}$ & $33.7^{b} \mathrm{C}$ & $29.5_{B}^{b}$ & $26.3_{A}^{b}$ & $33.6_{B}^{b}$ & $30.2_{\mathrm{A}}^{\mathrm{b}}$ \\
\hline South & $44.5^{\mathrm{d}} \mathrm{D}$ & $42.0^{\mathrm{e}} \mathrm{C}$ & $39.2^{\mathrm{e}} \mathrm{B}$ & $36.0_{A}^{\dagger}$ & $42.1_{\mathrm{B}}^{\mathrm{t}}$ & $38.8_{\mathrm{A}}^{\dagger^{n}}$ \\
\hline East & $36.8^{\mathrm{a}}$ & $32.3^{\mathrm{a}} \mathrm{c}$ & $27.7^{\mathrm{a}}$ & $24.6^{\mathrm{a}}{ }_{\mathrm{A}}$ & $31.9^{\mathrm{a}}$ & $28.7^{\mathrm{a}} \mathrm{A}$ \\
\hline West & $41.4^{\mathrm{C}} \mathrm{D}$ & $38.9^{d} \mathrm{C}$ & $36.2^{\mathrm{d}^{\mathrm{d}}}$ & $33.6^{e}{ }_{A}$ & $39.2^{\mathrm{e}} \mathrm{B}$ & $35.9^{e}{ }_{A}$ \\
\hline NE & $38.8^{\mathrm{b}}$ & $36.2^{\mathrm{c}} \mathrm{C}$ & $31.8^{\mathrm{C}}$ & $27.7^{\mathrm{C}}{ }_{\mathrm{A}}$ & $35.4^{\mathrm{C}}$ & $31.9^{C_{A}}$ \\
\hline NW & $40.7^{\mathrm{C}} \mathrm{D}$ & $38.1^{\mathrm{c}} \mathrm{C}$ & $35.1^{\mathrm{d}} \mathrm{B}$ & $31.6^{d_{A}}$ & $38.0^{\mathrm{c}}{ }_{\mathrm{B}}$ & $34.8^{d_{A}}$ \\
\hline SE & $43.8^{\mathrm{d}} \mathrm{D}$ & $44.6_{C}^{\dagger}$ & $41.5_{B}^{\dagger}$ & $38.4^{g^{n}}$ & $42.9^{g^{\circ}}$ & $41.3^{g^{n}}$ \\
\hline SW & $48.7^{e}$ & $46.5^{\mathrm{g}} \mathrm{C}$ & $43.6^{g_{B}}$ & $40.9^{h}{ }_{A}$ & $46.5^{\mathrm{n}^{\mathrm{b}}}$ & $43.3^{\mathrm{h}_{\mathrm{A}}}$ \\
\hline AXB & SEm \pm 0.4 & $-5 \% \quad 1.17$ & & & SEm \pm 0.29 & $\% \quad 0.83$ \\
\hline
\end{tabular}

The calcium carbonate content in soils ranged from 31.9 to $46.5 \mathrm{~g} \mathrm{~kg}^{-1}$ and 28.7 to $43.3 \mathrm{~g}$ $\mathrm{kg}^{-1}$ atsurface and sub-surface soil in relation to east to southwest wind directions, respectively. The calcium carbonate content increased with increasing distance from industries site and higher content at $0.5 \mathrm{~km}$ distances in surface soil in relation to southwest wind direction as compared to east wind directions. The higher deposition of cement dust nearer at the distance of $1 \mathrm{~km}$ may be due to increase in concentration of lime and $\mathrm{Ca}^{++}$mica the soil alkaline. Similar finding was reported by Khampariaet al. (2012).

From the results it may be concluded that soil $\mathrm{pH}, \mathrm{EC}$ and calcium carbonateincresed with decreasing distances from the cement industries 
at $0.5 \mathrm{~km}$ in surface soil in the southwest wind direction. Organic carbon decreased with decreasing in the distances from the cement

\section{REFERENCES}

Ahiamadjie, H., Adukpo, O. K., Tandoh, J. B. (2011) Determination of the elemental contents in soils around diamond cement factory, Aflao, Research. Journal of Environment and Earth Science 3(1): 4650.

Asadu, C. L. and Agada, C. (2008The impact of cement kiln dust on soil physico-chemical properties at Gboko, East Central Nigeria. Nigerian Journal of soil and Environment Research 8: 1595-6121.

Gbadebo, A. M. Bankole, O. D. (2007) Analysis of potential toxic metals in airborne cement dust around Sagamu, Southwestern Nigeria, Journal of Applied Science 7 (1): 5-40.

Grantz, D. A., Garner, J. H. and Johnson, D. W. (2003) Ecological effects of particulate matter. Environment Interaction 29: 213239.

Gupta, S. and Sharma, S. (2012) Compound effect of cement dust emitted by closely located cement plants on soil of nokha (Bikaner). Oriental Journal of Chemistry 28(4): 1919-1925.

Jackson, M. L. (1973) Soil Chemical Analysis. Prentice Hall of India Private Limited, New Delhi.

Khamparia, A., Chattergee, S. K. and Sharma, G. D. (2012) Assessment on effect of cement dust pollution on soil health. industries at $3 \mathrm{~km}$ in surface soil in the southwest wind direction.

Journal Environment Research Development 7 (1): 368-374.

Mlitan, A. B., Alajtal, A. I. and, Alsadawy, A. M. (2013) Toxicity of heavy metals and microbial analysis of soil samples collected from the area around zliten cement factory. Open Journal of Air Pollution 2:25-28.

Ocak, I. and Nowak, D. J. (2004)The effect of cement dust emitted from gaziantep cement plant on micro fungus flora of surroundings soils, Turkey. Trakya University Journal Science 5: 107-115.

Pandey, D. D. and Kumar, S. (2011) Impact of cement dust pollution on biomass chlorophyll and nutrients of plants. Environment Ecology 14: 872-875

Raajasubramanian, D., Hanumappa, K. R. and Kuppan, N. (2015). The study of change in physico-chemical properties of soil due to cement dust pollution-and hazardous terrorization to ecosystem. Canadian Journal of Pure and Applied Science 9(1): 3193-3200.

Raajasubramanian, D., Sundaramoorthy, P., Baskaran, L., Ganesh, K. S., Chidambaram, A. A. and Jeganathan, M. (2011) Cement dust pollution on growth and yield attributes of groundnut (ArachishypogaeaL.). International Multidisciplinary Research Journal 1(1): 31-36. 